\title{
Effect of an unrelated fluent action on word recognition: A case of motor discrepancy
}

\author{
Denis Brouillet $^{1} \cdot$ Audrey Milhau ${ }^{1} \cdot$ Thibaut Brouillet $^{2} \cdot$ Philippe Servajean $^{1}$
}

Published online: 9 September 2016

(C) Psychonomic Society, Inc. 2016

\begin{abstract}
It is now well established that motor fluency affects cognitive processes, including memory. In two experiments participants learned a list of words and then performed a recognition task. The original feature of our procedure is that before judging the words they had to perform a fluent gesture (i.e., typing a letter dyad). The dyads comprised letters located on either the right or left side of the keyboard. Participants typed dyads with their right or left index finger; the required movement was either very small (dyad composed of adjacent letters, Experiment 1) or slightly larger (dyad composed of letters separated by one key, experiment 2). The results show that when the gesture was performed in the ipsilateral space the probability of recognizing a word increased (to a lesser extent it is the same with the dominant hand, experiment 2). Moreover, a binary regression logistic highlighted that the probability of recognizing a word was proportional to the speed by which the gesture was performed. These results are discussed in terms of a feeling of familiarity emerging from motor discrepancy.
\end{abstract}

\section{Keywords Memory $\cdot$ Fluency $\cdot$ Discrepancy $\cdot$ Gesture}

The way people conceive the world is intrinsically dependent on body specificity and on the way they interact with their environment (Casasanto, 2009). The aim of this study was to extend previous findings (i.e., typing activity) by showing that

Denis Brouillet

denis.brouillet@univ-montp3.fr

1 EPSYLON Laboratory Site St Charles, University Paul Valéry, Route de Mende, 34199 Montpellier CEDEX 5, France

2 CERSM Laboratory, University Paris X, Nanterre, France recognition judgment could be influenced by the hand one uses and the space in which a response is given.

Manual dominance is one of the main body specificities. The results observed in studies of typing activity showed that skilled typists on the QWERTY keyboard preferred letters typed with the right hand to those typed with the left hand. But when participants were asked to perform the preference task while holding a motor plan in memory (i.e., dual task), the skilled typists' preference was attenuated when the motor plan involved the same finger used to type the presented dyads (see Beilock \& Holt, 2007). Jasmin and Casasanto (2012) confirmed that preference judgments are influenced by motor fluency caused by digit-specific typing simulation (the so-called QWERTY effect). Yang, Gallo, and Beilock (2009) showed that the fluency with which participants type the letter dyads to be recognized influences their recognition judgment. Participants found it easier to remember letter dyads typed with two different fingers than those typed with one finger. Indeed, typing a letter dyad with two different fingers is easier than typing a letter dyad with one finger.

If manual dominance is one of the main body specificities, laterality is another one: an individual's most fluent actions are those executed with the dominant hand on the dominant side (i.e., for a right-hander, movements of the right hand on the right side). Classic studies from aim-pointing tasks have already demonstrated that ipsilateral actions are carried out more easily and faster than contralateral actions for both the dominant and the non-dominant hand (Fisk \& Goodale, 1985). In other words, independently of handedness, the right hand should be more fluent on the right side of space than on the left part, and the left hand should be more fluent on the left part than on the right part of space (de la Vega et al., 2013). Moreover, Milhau, Brouillet, and Brouillet (2013, 2014) showed that it was necessary to take into account the effect of the location of responses, by considering their congruency 
with the motor fluency experienced during the task rather than the motor fluency corresponding to the participant's handedness.

All these studies support the ideas that motor compatibilities generate fluency (Brouillet, Ferrier, Grosselin, \& Brouillet, 2011; Cannon, Hayes, \& Tipper, 2010) and that the more fluently an item is processed the more it is judged to be familiar (Susser \& Mulligan, 2015; Topolinski, 2012; Topolinski \& Strack, 2009, 2010; Whittlesea, 1993). However, Whittlesea and Williams (1998) argued that a feeling of familiarity can also occur when the way the current treatment takes place is surprising, what they call "discrepancy" (for a review see Leboe-MacGowan \& Whittlesea, 2013). For example, Whittlesea and Williams (2001, Experiment 1) noted that presenting words at the end of predictive sentences did not lead to creating a false alarm when the sentence stem and the terminal word were presented simultaneously but when there was a brief pause between the sentence stem and the occurrence of the terminal word (e.g., "The stormy seas tossed the ... boat"). These results show that fluency is not directly responsible for the feeling of familiarity, but it is the tension arising from expectation linked to the pause (i.e., what word will follow?) that generates discrepancy. In turn as one cannot attribute this discrepancy to its source people attribute it to the most obvious source: prior word exposure. This unconscious source of attribution results in a conscious feeling of familiarity.

Traditionally, in a recognition task (i.e., is this word present or not in the learning phase?) participants have to perform a gesture to give their response: press a touch on a keyboard. The touch "yes" (or «not») is on the right or on the left of the keyboard, and the participants give their response with their right hand or their left hand or with both. The question is whether the gesture performed to give the response can influence, by itself, the recognition judgment.

The aim of this study was to show that the fluency of a gesture (i.e., dominant hand and ipsilateral space) that has no ties with the meaning of the words to be judged, performed before the recognition response was given, can generate discrepancy and consequently familiarity. Indeed, we assume that after a fluent gesture participants experience a feeling of fluency whereas when the word to be recognized appears uncertainty exists with respect to the prior presentation of the word, which generates tension (see Whittlesea, 1993; Whittlesea \& Williams, 2001). Thus, it is reasonable to hypothesize that after a fluent gesture that has no ties with the meaning of the words to be judged this word will be more likely recognized than a word following a non-fluent gesture.

The difference between our study and those described above is in the distinction made by Leboe-MacGowan and Whittlesea (2013) between absolute fluency (i.e., motor fluency was associated with motor memory associated with the words, as in the studies described above) and relative fluency (i.e., motor fluency was not associated with the words to be recognized but associated with a subtask, as in our study).

\section{Experiment 1}

We expected that the recognition rate would be higher when the action that preceded a recognition judgment was performed with the dominant hand than when it was performed with the non-dominant hand. We also expected that words judged after an action performed in ipsilateral space would be more likely to be recognized than words judged after a contralateral action. According to Milhau, Brouillet, and Brouillet (2014), we expected an interaction between hand and laterality.

\section{Method}

Participants Forty volunteer students from the University of Montpellier participated in this experiment; all were righthanded. They were randomly assigned to one of two groups (right-hand responding; left-hand responding). Each group consisted of 12 women and eight men. The age of participants ranged from 19 to 24 years (mean $=20.3$ years; $S D=2.03$ ). All were native French speakers, had normal or corrected-tonormal vision, and were right-handed. All participants gave written informed consent to participate in the study.

Materials We selected 48 neutral words from a French standard list of emotional words (Bonin et al., 2003). The words in the standard list are rated on a 1 (low) to 5 (high) scale with respect to several characteristics. The 48 words we selected were between 5 and 8 letters long, and had mean scores of 4.68 for concreteness $(S D=0.67), 4.21$ for imagery $(S D=$ $1.03), 3.15$ for frequency $(S D=0.90)$, and 3.06 for valence $(S D=0.87)$.

We used a Logitech MK240 AZERTY keyboard which had been modified so that none of the keys was in its original location. This reorganization of the keys allowed us to control for potential effects of keyboard expertise insofar as the arrangement of our keyboard was unfamiliar to all participants. In addition our modified keyboard had the same number of keys on the right side as a normal keyboard. The keys $\mathrm{Y}, \mathrm{U}, \mathrm{I}, \mathrm{O}, \mathrm{P}, \mathrm{H}, \mathrm{J}, \mathrm{K}, \mathrm{L}, \mathrm{M}, \mathrm{B}, \mathrm{N}$, which are to the right of the median axis in the conventional AZERTY layout, were replaced by the keys T, R, E, Z, A, G, F, D, S, Q, V, C, which are to the left of the median axis in the conventional layout. The assignment of the keys was based on a random draw. The median axis (keys Y, H, B in the conventional layout) was indicated with the keys $>$, ?, \%. The 12 letter keys on each side of our modified layout were spread over three rows, giving four keys per row. The layout of the modified keyboard used was as follows, right side, top row: S, G, C, Q, middle 
row: A, T, D, V, bottom row: R, E, Z, F; left side, top row: B, J, $\mathrm{M}, \mathrm{H}$, middle row: $\mathrm{U}, \mathrm{N}, \mathrm{O}, \mathrm{I}$, bottom row: $\mathrm{Y}, \mathrm{K}, \mathrm{P}, \mathrm{L}$.

The stimuli for the typing task consisted of 48 letter dyads; 24 had to be typed on the right side of the keyboard, 24 on the left side. In all dyads the first letter to be typed corresponded to a key closer to the median axis than the second letter. The letters of a dyad corresponded to keys positioned adjacent to one another on the horizontal axis (e.g., S-G; B-J) or the diagonal axis (e.g., T-C; $\mathrm{K}-\mathrm{O}$ ). This gave us 30 different dyads (nine horizontal dyads and six diagonal dyads on each side); to make a set of 48 dyads we repeated the $9 \times 2$ horizontal dyads. The order of presentation of the 48 dyads was randomized for each participant and the letters of the dyads were displayed in black 28-point Times font.

Procedure The experiment was programmed and run using E-prime software (Schneider et al., 2002). Participants were seated $60 \mathrm{~cm}$ from the computer screen (17-in.). Participants were tested individually in an isolated room by a single experimenter. Each experimental session started with a brief description of the task to be performed after which the participant provided consent to participate. After consent had been given the experiment started; it was divided into two phases: a learning phase and a recognition phase.

Learning phase In the learning phase participants were shown a list of 24 words and were explicitly informed that they would subsequently be tested on their memory for them. First a fixation cross was displayed in the center of the screen for $500 \mathrm{~ms}$. Then a word was presented on the screen for $200 \mathrm{~ms}$ in black 28-point Times font. Presentation of a word was followed by presentation of a blank screen for $500 \mathrm{~ms}$. The order of presentation of words was randomized.

Recognition phase We used a modification of the standard recognition task procedure. Before test words were presented the participant was required to type the two letters that appeared in succession in the center of the screen on the computer keyboard (typing task). Each letter remained on the screen until it had been typed. Only after the second letters had been typed were the test words displayed in the center of the computer screen. The participant was then asked to indicate verbally whether the word had been included in the learning list (recognition task). Participants gave their recognition judgment verbally rather than via a motor action (pressing a computer key), as in the conventional procedure, in order to avoid motor interference. Each trial started with a black fixation cross which remained in the center of the gray screen for $500 \mathrm{~ms}$.

Participants were told to type as quickly and accurately as possible by pressing the appropriate keys on the keyboard. Participants were instructed to use their right index finger (right-hand responders) or their left index finger (left-hand responders) to type the letters; they were required to use a single finger to type both letters in order to ensure that the typing response involved a leftward or rightward response. Participants were instructed to place their response finger on the cross drawn on the space bar at the start of a trial and to return it to this position after typing.

In the recognition task participants were shown a randomized list of 48 words. Half the list was made up of the 24 words presented in the learning task (old words); the other half had not been presented in the learning task (NEW words). To prevent a potential word effect, the old word for a participant became the new word for another participant, and reciprocally, the new word for a participant became the old one for another. The old and new words were matched in terms of length, concreteness, imagery, frequency, and valence $(t(38)<1)$. The words were displayed in black 28-point Times font and appeared automatically in the center of the screen $250 \mathrm{~ms}$ after the second letter of a dyad had been typed (see Whittlesea, 2002 for the rationale for an interval been typing response and recognition stimulus presentation); words were displayed for 1,000 ms. Participants were instructed to respond verbally, as quickly and accurately as possible, indicating whether the word had or had not been presented in the learning phase. The experimenter noted the participant's response on an answer sheet.

In total, we investigated three sources of variance: the status of the words (old vs. new) and the laterality of the typing response (ipsilateral vs. contralateral) were within-participants factors; the hand used to execute the typing response (right vs. left) was a between-participants factor. All other variations were controlled variables.

\section{Results}

First we present data on time taken to type the second letter of the dyads, after the first letter was typed. ${ }^{1}$ That is to say, the time that elapsed from the onset of the second letter until the corresponding key had been pressed (Table 1). This time can be interpreted as an indication of the fluency of the gesture made to type the second letter of the dyads. Thereafterwe present data on recognition scores (Table 2). We got less than $1 \%$ error and the trials in which typing responses were incorrect were excluded from analyses of typing speed and recognition judgment.

Fluency Statistical analyses revealed that the hand used to type made no difference to the speed of typing responses, $F$ $<1$. There was an effect of laterality; participants typed faster on the ipsilateral side than on the contralateral side, $F(1,38)=$

\footnotetext{
${ }^{1}$ The statistical analysis on the time for typing the first letter revealed no difference for the hand used, nor for laterality, and no interaction, $\mathrm{F}<1$.
} 
Table 1 Mean response times (in ms) organized by hand (left; right) and response laterality (contralateral; ipsilateral). SE is presented in brackets

\begin{tabular}{lll}
\hline & Contralateral action & Ipsilateral action \\
\hline Left hand & $581.3(164.46)$ & $529.4(103.9)$ \\
Right hand & $567.5(90.75)$ & $522.6(100.3)$ \\
\hline
\end{tabular}

$4.22, p=.04, \eta_{\mathrm{p}}^{2}=.10$. There was no interaction between the hand used and laterality, $F<1$.

Recognition Statistical analyses showed an effect of word status; participants judged old words more frequently to be old than new words, $F(1,38)=703.32, p<.001, \eta_{\mathrm{p}}^{2}=.94$. There was also an effect of laterality; participants were more likely to report that they recognized a word which was preceded by an ipsilateral typing response than one preceded by a contralateral typing response, $F(1,38)=7.07, p=.011, \eta_{\mathrm{p}}^{2}=$ .15 . None of the other factors affected recognition.

\section{Discussion}

As expected it is easier to type letters on the ipsilateral side than on the contralateral side, and this facility was accompanied by a higher recognition rate without affecting the ability to discriminate correctly between old and new words. In other words, regardless of the hand used, executing an action on the ipsilateral side affects recognition judgment even if the action is not directly linked to the meaning of the words being judged.

As these results suggest that the probability of responding "old" is associated with the fluency of the gesture, we analyzed the relationship between the response time (an indication of the fluency of the gesture) in a given trial and the likelihood of judging the item in the given trial to be old. To do that, we used a binary logistic regression to model the dichotomous outcome variable "subject's response." The observation of each $i$ subject at each $t$ time of measurement is treated as a realization of a Bernoulli random variable that takes the value 1 (i.e., response "old") with success probability of $\mathrm{P}_{i t}$. Our objective was to estimate (standardized regression coefficient) the change in the Logit of the probability $\mathrm{P}_{i t}$ with a

Table 2 Mean probability to judge words as old organized by response hand (left; right), word status (OLD; NEW), and response laterality (contralateral; ipsilateral)

\begin{tabular}{llllll}
\hline & \multicolumn{2}{l}{ Left hand } & & \multicolumn{2}{l}{ Right hand } \\
\cline { 2 - 3 } & OLD & NEW & & OLD & NEW \\
\hline Contralateral & 0.79 & 0.14 & & 0.74 & 0.13 \\
Ipsilateral & 0.79 & 0.17 & & 0.79 & 0.18 \\
\hline
\end{tabular}

unit change in the predictor "Time Response." Our results showed that the probability of recognizing one item declined as much as the value of the TR increased $(B=-.36 ; \mathrm{p}<.01)$.

Despite the fact that this result supports our hypothesis, we did not find a dominant hand effect on typing fluency. We believe that the lack of effect of dominant hand is related to the very small amplitude of the movement required to type the second letter in the dyads (letters were adjacent on the keyboard). In fact the amplitude was not sufficient to produce a detectable difference in the fluency of movements executed with the dominant and non-dominant hands in our righthanded sample. To address this problem we conducted a second experiment in which the letters of the dyads were spaced further apart on the keyboard.

\section{Experiment 2}

\section{Method}

Participants Forty volunteer students from the University of Montpellier who had not taken part in Experiment 1 participated in this experiment; all were right-handed. They were randomly assigned to the groups (right-hand responding; left-hand responding). Both groups consisted of 14 women and six men aged between 20 and 24 years (mean $=20.8$ years; $S D=2.23$ ). All participants were native French speakers, had normal or corrected-to-normal vision, and were right-handed. All participants gave written informed consent to participate in the study.

Materials We used the same 48 words and the same modified keyboard as in Experiment 1.

Procedure The experiment was divided into two phases: a learning phase and a recognition phase.

Learning phase The procedure was the same as in Experiment 1. Participants were shown a list of 24 words and were explicitly informed that they would subsequently be tested on their ability to remember them.

Recognition phase The procedure was the same as in Experiment 1, except that in the typing task the letters for the dyads were separated by one key, rather than being adjacent (e.g., horizontal dyads: S-C, B-M; diagonal dyads: T-Q, $\mathrm{K}-\mathrm{I})$. This constraint meant that only 12 different dyads were possible (six horizontal dyads; six diagonal dyads), so each dyad was repeated four times.

In total, we investigated three sources of variance: the status of the words (OLD vs. NEW); and the laterality of the typing response (ipsilateral vs. contralateral) were withinparticipants factors; the hand used to execute the typing 
Table 3 Mean response times (in ms) organized by hand used (left; right) and response laterality (contralateral; ipsilateral). SE is presented in brackets

\begin{tabular}{lll}
\hline & Contralateral action & Ipsilateral action \\
\hline Left hand & $814,3(119,3)$ & $707,5(118,2)$ \\
Right hand & $785,2(115,1)$ & $670,6(126,1)$ \\
\hline
\end{tabular}

response (right vs. left) was a between-participants factor. All other variations were controlled variables.

\section{Results}

First we present data on the time taken to type the second letter of the dyads ${ }^{2}$ (Table 3 ) and then we present the recognition scores (Table 4). We got less than $1 \%$ error and the trials in which typing responses were incorrect were excluded from analyses of typing speed and recognition judgment.

Fluency Statistical analysis revealed that participants typed the letters at a similar speed regardless of whether they were using their right or left hand, $F<1$. There was, however, an effect of laterality; participants typed the letters faster on the ipsilateral side than on the contralateral side, $F(1,38)=25.34, p<.001, \eta_{p}^{2}=.40$. Crucially, there was a hand-by-laterality interaction, $F(1,38)=5.45, p=.02, \eta_{\mathrm{p}}^{2}=.12$. Partial analysis showed that when typing responses were executed on the ipsilateral side there was a marginal difference between the speed of left- and right-hand responses, $F(1,38)=3.68, p=.06, \eta_{\mathrm{p}}^{2}=.08$. Participants using their right hand tended to be faster than those using their left hand. There was also a marginal difference between right- and left-hand typing speeds for contralateral typing responses, $F(1,38)=3.36, p=.07, \eta_{\mathrm{p}}^{2}=$ .08; participants using their right hand tended to be faster than those using their left hand. Partial analysis also revealed that amongst left-hand responders there was a marginal difference between the speeds of ipsilateral and contralateral typing responses, $F(1,38)=3.58$, $p=.06, \eta_{\mathrm{p}}^{2}=.08$; amongst right-hand responders this difference reached significance, $F(1,38)=27.29, p<$ $.001, \eta_{\mathrm{p}}^{2}=.41$. In summary the analyses suggested that participants were faster when typing on the ipsilateral side than on the contralateral side, particularly if they were using their right hand.

Recognition Statistical analysis showed an effect of word status; participants judged old words to be old rather than new words, $F(1,38)=643.21, p<.001, \eta_{p}^{2}=.94$. There

\footnotetext{
$\overline{2}$ The statistical analysis on the time for typing the first letter revealed no difference for the hand used, nor for laterality, and no interaction, $\mathrm{F}<1$.
}

Table 4 Mean probability to judge words as "old" organized by response hand (left; right), word status (old; new), and response laterality (contralateral; ipsilateral)

\begin{tabular}{llllll}
\hline & \multicolumn{2}{l}{ Left hand } & & \multicolumn{2}{l}{ Right hand } \\
\cline { 2 - 3 } \cline { 5 - 6 } & OLD & NEW & & OLD & NEW \\
\hline Contralateral & 0.64 & 0.09 & & 0.68 & 0.12 \\
Ipsilateral & 0.79 & 0.40 & & 0.85 & 0.46 \\
\hline
\end{tabular}

was also an effect of hand: participants were more likely to report recognition of test words if they were typing the preceding letter dyad with their right hand rather than with their left hand, $F(1,38)=7.98, p<.01, \eta_{p}^{2}=.17$. There was also an effect of laterality: participants were more likely to report recognition of words preceded by an ipsilateral typing response than words preceded by a contralateral typing response, $F(1,38)=112.12, p<.001, \eta_{p}^{2}=.74$. However, there was no interaction between hand and laterality. There was a status-by-laterality interaction, $F(1,38)=12.76, p<.001, \eta_{\mathrm{p}}^{2}=.25$. Partial analysis showed that the probability to judge words as "old" were higher when the test word was preceded by an ipsilateral typing response than when it was preceded by a contralateral typing response, for both old and new words, but more accentuated for new; OLD: $F(1,38)=19.01, p<.001, \eta_{p}^{2}=.33 ;$ NEW: $F(1,38)$ $=125.56, p<.001, \eta_{\mathrm{p}}^{2}=.76$. There were no other interactions.

As in Experiment 1, we analyzed the relationship between the response time in a given trial and the likelihood of judging the item in the given trial to be old. Our results showed that the probability of recognizing one item declined as much as the value of the TR increased $(\mathrm{B}=-.56 ; \mathrm{p}<.0001)$.

\section{Conclusion}

Experiment 2 confirmed the effects of laterality observed in Experiment 1: Participants typed faster and recognized more words when typing on the ipsilateral side than when typing on the contralateral side. As expected, increasing the amplitude of the gesture required in the typing response resulted in: (a) a detectable difference in the speed of ipsilateral responses: Typing was faster with the right hand than with the left hand; (b) a higher probability to recognize words with the right hand than the left hand. Even if there was no interaction between hand and laterality, we can observe that the probability to recognize words is the highest when the gesture was performed with the right hand in the ipsilateral space. Finally, we observed that the probability of recognizing a word 
increased as much the time to type the letters that precedes the onset of this word decreased.

\section{General discussion}

The aim of this study was to extend findings that showed that motor fluency gives rise to a feeling of familiarity resulting in a judgment of recognition (e.g., Yang, Gallo, \& Beilock, 2009; Susser \& Mulligan, 2015). Contrary to these works, in our studies motor fluency was not associated with the words to be recognized but associated with a subtask. We manipulated the fluency of a gesture that has no ties with the meaning of the words to be recognized, performed before the recognition judgment. According to Whittlesea and Williams (2001), this situation should generate discrepancy (i.e., feeling fluency and incertitude with respect to prior presentation) and consequently familiarity, what we call a situation of "motor discrepancy." So we hypothesized that the probability of recognizing a word should be associated with the fluency of the gesture performed before the memory judgment.

The results are consistent with our hypothesis. They showed firstly that ipsilateral side responses were easier to execute than contralateral responses, and secondly that words that followed an ipsilateral response were more likely to be reported as recognized than words that followed a contralateral response. An effect of use of the dominant hand was shown when increasing the amplitude of the motor responses. Broadly, the probability of recognizing a word is proportional to the speed of the gesture performed.

These findings corroborate a key tenet of the Selective Construction And Preservation of Experience (SCAPE) model: “... extra-fluent processing is (simply) experienced as feeling of familiarity" (Whittlesea, 1997, p. 244). More specifically, they highlight the relevance of the concept of discrepancy proposed by Whittlesea and Williams (1998). Typing responses that involve an ipsilateral gesture generated a sensation of fluency. However, participants cannot attribute this subjective feeling to the gesture performed because they are not conscious of this phenomenon. When a test word appears on the screen participants are initially uncertain as to whether it was on the learning list. Thus this discrepancy (i.e., feeling fluency and incertitude with respect to prior presentation) generates a non-specific signal automatically triggering a search for some explanation. As participants cannot firmly attribute this non-specific signal to its source, "they resolve this discrepancy by attributing it to the most salient source available. As their current task requires making recognition judgments, prior stimulus exposure is the most obvious cause of the discrepancy" (Leboe-MacGowan \& Whittlesea, 2013 , p. 256) and thus tend to conclude that the word was on the learning list and report that they recognize it. Statistical analysis of word status effects support this view: Performing a fluent gesture before giving a recognition judgment had more effect on the probability to judge new words as "old," than old ones, indicating that recognition judgments are influenced by the sensation of fluency and the feeling of familiarity associated, regardless of its source (note that the performance of a fluent gesture also affects the probability to judge old words as "old," which excluded an interference effect from the task of tapping). But mainly, it is the relationship between the time of typing the letters and the subject's response that support the discrepancy hypothesis.

One might conclude that a fluent gesture executed before the recognition response was given affects memory judgment and that this finding should be taken into account when assessing performance on recognition tasks, as in other computer-controlled tasks.

\section{References}

Beilock, S. L., \& Holt, L. E. (2007). Embodied preference judgments: Can likeability be driven by the motor system? Psychological Science, 18, 51-57.

Bonin, P., Méot, A., Aubert, L., Malardier, N., Niedenthal, P., \& CapelleToczek, M. C. (2003). Normes de concrétude, de valeur d'imagerie, de fréquence subjective et de valence émotionnelle pour 866 mots. L'année Psychologique, 103(4), 655-694.

Brouillet, T., Ferrier, L. P., Grosselin, A., \& Brouillet, D. (2011). Action compatibility effects are hedonically marked and have incidental consequences on affective judgment. Emotion, 11(5), 1202.

Cannon, P. R., Hayes, A. E., \& Tipper, S. P. (2010). Sensorimotor fluency influences affect: Evidence from electromyography. Cognition \& Emotion, 24(4), 681-691.

Casasanto, D. (2009). Embodiment of Abstract Concepts: Good and Bad in Right- and Left-Handers. Journal of Experimental Psychology: General, 138(3), 351-367.

de la Vega, I., Dudschig, C., De Filippis, M., Lachmair, M., \& Kaup, B. (2013). Keep your hands crossed: The valence-by-left/right interaction is related to hand, not side, in an incongruent hand-response key assignment. Acta Psychologica, 142(2), 273-277.

Fisk, J. D., \& Goodale, M. A. (1985). The organization of eye and limb movements during unrestricted reaching to targets in contralateral and ipsilateral visual space. Experimental Brain Research, 60(1), 159-178.

Jasmin, K., \& Casasanto, D. (2012). The QWERTY effect: How typing shapes the meanings of words. Psychonomic Bulletin and Review, 19(3), 499-504.

Leboe-McGowan, J. P., \& Whittlesea, B. W. A. (2013). Through the SCAPE looking-glass: Sources of performance and sources of attribution. In D. Reisberg (Ed.), Handbook of Cognitive Psychology. New York: Oxford University Press.

Milhau, A., Brouillet, T., \& Brouillet, D. (2013). Biases in evaluation of neutral words due to motor compatibility effect. Acta psychologica, 144(2), 243-249.

Milhau, A., Brouillet, T., \& Brouillet, D. (2014). Valence-space compatibility effects depend on situated motor fluency in both right- and left-handers. Quarterly Journal of Experimental Psychology, 68(5), $1-13$.

Schneider, W., Eschman, A. \& Zuccolotto, A. (2002). E-Prime User's Guide. Pittsburgh: Psychology Software Tools Inc. 
Susser, J. A., \& Mulligan, N. W. (2015). The effect of motoric fluency on metamemory. Psychonomic bulletin \& review, 22(4), 1014-1019.

Topolinski, S. (2012). The sensorimotor contributions to implicit memory, familiarity, and recollection. Journal of Experimental Psychology: General, 141(2), 260.

Topolinski, S., \& Strack, F. (2009). Motormouth: Mere exposure depends on stimulus-specific motor simulations. Journal of Experimental Psychology: Learning, Memory, and Cognition, 35(2), 423.

Topolinski, S., \& Strack, F. (2010). False fame prevented: avoiding fluency effects without judgmental correction. Journal of personality and social psychology, 98(5), 721.

Whittlesea, B. W. (1993). Illusions of familiarity. Journal of Experimental Psychology: Learning, Memory, and Cognition, 19(6), 1235.

Whittlesea, B. W. A. (1997). Production, evaluation and preservation of experiences: Constructive processing in remembering and performance tasks. In D. L. Medin (Ed.), The psychology of learning and motivation (Vol. 37, pp. 211-264). New York: Academic Press.

Whittlesea, B. W. A. (2002). Two routes to remembering (and another to remembering not). Journal of Experimental Psychology: General, 131(3), 325-348.

Whittlesea, B. W. A., \& Williams, L. D. (1998). Why do strangers feel familiar, but friends don't? A discrepancy-attribution account of feelings of familiarity. Acta Psychologica, 98(2-3), 141-165.

Whittlesea, B. W., \& Williams, L. D. (2001). The discrepancy-attribution hypothesis: II. Expectation, uncertainty, surprise, and feelings of familiarity. Journal of Experimental Psychology: Learning, Memory, and Cognition, 27(1), 14.

Yang, S., Gallo, D., \& Beilock, S. L. (2009). Embodied memory judgments: A case of motor fluency. Journal of Experiment Psychology: Learning, Memory, \& Cognition, 35, 1359-1365. 